ence group $G-F(G)$ which contains properly the difference group $G^{\prime}-F^{\prime}(G)$ since $F^{\prime}(G)=F(G)$. Now $I^{\prime}(G)$ is isomorphic to $G^{\prime}-F^{\prime}(G)$ and to $I(G)$. Hence $G-F(G)$ is an $I$-group and it follows that $I(G)$ which is isomorphic to $G-F(G)$ is also an $I$-group.

It follows from Theorem 7 that the theorems and corollaries of $\$ \$ 2$ and 3 survey completely all completely reducible groups, $G$, which are $I$-groups.

UNIVERSITY OF WASHINGTON

\title{
AN EXISTENCE THEOREM FOR LATIN SQUARES
}

\section{MARSHALL HALL}

1. Introduction. A latin square may be interpreted as a representation of a 3-web or as the multiplication table of a quasi-group. Hence the following theorem has application both in the theory of projective planes and in the theory of quasi-groups. It is derived from a very interesting result of $\mathrm{P}$. Hall.

2. The existence theorem. Is there any combinatorial restriction which prevents us from constructing a latin square by adding a row at a time? The following theorem shows that such a procedure is permissible.

THEOREM. Given a rectangle of $n-r$ rows and $n$ columns such that each of the numbers $1,2, \cdots, n$ occurs once in every row and no number occurs twice in any column, then there exist $r$ rows which may be added to the given rectangle to form a latin square.

Proof. Let $C_{i}, i=1,2, \cdots, n$ be the subset of the numbers $1,2, \cdots, n$ which do not occur in the $i$ th column of the given rectangle. Then each $C_{i}$ contains $r$ numbers and each number occurs $r$ times in all the $C$ 's. For there are $n-r$ numbers in the $i$ th column and each number has appeared in $n-r$ columns. It will be shown that the subsets satisfy the requirements of P. Hall's theorem $:^{1}$

In order that a complete system of distinct representatives of subsets

Received by the editors January 19, 1945.

Opinions and assertions in this paper are those of the author and are not to be construed as official or as reflecting the views of the United States Navy Department or the Naval Service at large. 30.

${ }^{1}$ P. Hall, On representatives of subsets, J. London Math. Soc. vol. 10 (1935) pp. 26- 
$T_{1}, \cdots, T_{m}$ of a set $S$ shall exist, it is sufficient that for each $k=1, \cdots, m$ any selection of $k$ of the subsets shall contain between them at least $k$ elements of $S$.

The necessity of these requirements is evident. Let us apply thitheorem to the subsets $C_{i}$. Any selection of $k C$ 's will contain $k r$ numbers and at least $k$ of these must be distinct since each number is contained in only $r C^{\prime}$ 's. The distinct representatives $c_{1}, \cdots, c_{n}$ of the subsets $C_{1}, \cdots, C_{n}$ may be added as a row to the given rectangle. For $c_{1}, \cdots, c_{n}$ must contain each of the numbers $1, \cdots, n$ once and no $c_{i}$ has appeared in the $i$ th column of the given $n-r$ rows. Repeatedly applying this process, we continue adding rows to the rectangle until it becomes a complete latin square.

United States Navy 